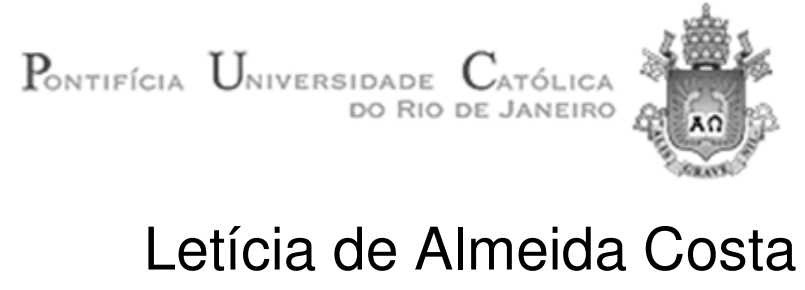

\title{
Avaliação Econômica do Projeto GTL: uma Aplicação da Teoria de Opções Reais com Processo de Reversão à Média
}

\author{
Dissertação de Mestrado \\ Departamento de Engenharia Industrial \\ Programa de Pós-Graduação em \\ Engenharia Industrial da PUC-Rio.
}

Orientador: Carlos Patrício Samanez Co-orientador: Marco Antonio Guimarães Dias

Rio de Janeiro

Março de 2007 


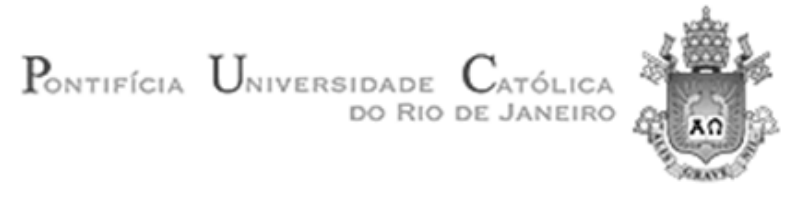

Letícia de Almeida Costa

\section{Avaliação Econômica do Projeto GTL: uma Aplicação da Teoria de Opções Reais com Processo de Reversão à Média}

Dissertação apresentada como requisito parcial para obtenção do título de Mestre pelo Programa de PósGraduação em Engenharia Industrial da PUC-Rio. Aprovada pela Comissão Examinadora abaixo assinada.

Prof. Carlos Patrício Samanez Orientador Departamento de Engenharia Industrial - PUC-Rio

Prof. Marco Antonio Guimarães Dias Departamento de Engenharia Industrial - PUC-Rio / Petrobras

Prof. José Paulo Teixeira Departamento de Engenharia Industrial - PUC-Rio

Prof. Paulo Henrique Soto Costa

Departamento de Engenharia Industrial - PUC-Rio

Prof. José Eugenio Leal

Coordenador (a) Setorial do Centro Técnico Científico - PUC-Rio 
Todos os direitos reservados. É proibida a reprodução total ou parcial do trabalho sem autorização da universidade, da autora e do orientador.

\section{Letícia de Almeida Costa}

Graduou-se em Ciências Econômicas pela PUC-Rio em Janeiro de 2002, onde foi monitora em diversas disciplinas. Durante o mestrado, devido ao seu rendimento acadêmico, foi beneficiada com bolsas de desempenho da PUC e participou do Projeto PUC/Petrobras de Análise de Investimentos usando a Teoria de Opções Reais.

Ficha Catalográfica

Costa, Letícia de Almeida

Avaliação Econômica do Projeto GTL: uma Aplicação da Teoria de Opções com Processo de Reversão à Média / Letícia de Almeida Costa; orientador: Carlos Patrício Samanez. - 2007.

147 f.; $30 \mathrm{~cm}$

Dissertação (Mestrado em Engenharia Industrial) Pontifícia Universidade Católica do Rio de Janeiro, Rio de Janeiro, 2007.

Incluí referências bibliográficas.

1. Engenharia Industrial - Teses. 2. Análise de investimento. 3. Teoria das Opções Reais. 4. Movimento de Reversão à Média. I. Samanez, Carlos Patrício. II. Pontifícia Universidade Católica do Rio de Janeiro. Departamento de Engenharia Industrial. III. Título.

CDD: 658.5 
À minha irmã, Aline, que me possibilitou ir atrás de um sonho e realizá-lo. Muito obrigada, amo você. "Um sonho que se sonha só é só um sonho. Mas, um sonho que se sonha junto, é realidade."

(Raul Seixas)

Aos meus pais, José e Marisa, que sempre estão do meu lado Me apoiando e incentivando em todos os momentos. Que alguma dia eu faça pelos meus filhos o que vocês fazem por mim. Amo muito vocês. 


\section{Agradecimentos}

À minha família pela torcida e pelo carinho que sempre tiveram comigo. Em especial a minha avó, Joaquina pelo apoio e paciência nos momentos finais dessa dissertação.

Ao Pedro, pelo amor, compreensão e força durante os anos do mestrado.

Aos professores Carlos Patrício e Marco Antônio, pelo estímulo, paciência, amizade e orientação que me ofereceram, sempre priorizando o meu aprendizado.

Aos professores José Paulo e Tara pelos ensinamentos dados sem os quais eu não conseguiria obter o conhecimento necessário para fazer este trabalho.

Ao professor Paulo Henrique e amigo Ivar Lira pela ajuda nas correções para a última versão deste trabalho.

Aos amigos e colegas de estudo, pela companhia e amizade nesses anos, em especial, Felipe, Marcela e Eduardo.

Ao Centro de Pesquisa da Petrobras (CENPES), em especial à equipe da Célula GTL pelo suporte financeiro e pela troca de informações e conhecimento transmitidos pelos profissionais que nos auxiliaram bastante, principalmente Luís Alberto Leite, Alessandro Oliveira, Sirlei Sousa e Henrique Cerqueira. E ao programador da Petrobras, Edison Tito, pela ajuda computacional fornecida.

À Coordenação de Aperfeiçoamento de Pessoal de Nível Superior (CAPES) e ao Departamento de Engenharia Industrial da PUC-Rio, pelos auxílios concedidos e pelo apoio financeiro. 


\section{Resumo}

Costa, Letícia de Almeida; Samanez, Carlos Patrício. Avaliação Econômica do Projeto GTL: uma Aplicação da Teoria de Opções Reais com Processo de Reversão à Média. Rio de Janeiro, 2007. 147p. Dissertação de Mestrado - Departamento de Engenharia Industrial, Pontifícia Universidade Católica do Rio de Janeiro.

O presente trabalho tem como objetivo analisar a viabilidade econômica de uma planta XTL usando a teoria das Opções Reais. Esta metodologia é a mais adequada para avaliar a capacidade que este tipo de projeto dispõe de trocar de input e/ou output, ou seja, eleger a cesta que maximiza o resultado final, de acordo com cada cenário. Essa política ótima permite avaliar a construção de uma planta com flexibilidades, com o investimento ocorrendo em um ambiente de incerteza, onde os preços (fatores de incerteza) serão considerados estocásticos e seguirão um Movimento de Reversão à Média. Os cálculos numéricos serão feitos através da simulação de Monte Carlo. A tecnologia, designada XTL, está dividida em duas etapas: um processo de gaseificação seguido de um processo GTL (gas-toliquid). A gaseificação permite transformar sólidos, líquidos e gases em gás de síntese, que será usado como input do GLT. Já o GTL possibilita transformar o gás de síntese em líquidos de alta qualidade, tais como nafta, diesel, parafinas e lubrificantes. Por associação, esta dissertação faz parte de um projeto que visa aplicar a teoria de Opções Reais na avaliação de investimentos em Pesquisa e Desenvolvimento (P\&D), com a finalidade de valorar e considerar as flexibilidades inerentes a este tipo de projeto.

\section{Palavras-chave}

Análise de Investimento, Teoria das Opções Reais, Movimento de Reversão à Média. 


\section{Abstract}

Costa, Letícia de Almeida; Samanez, Carlos Patrício. Valuation of GTL Project: a Real Option Aplication with Mean Reversion Model. Rio de Janeiro, 2007. 147p. MSc. Dissertation - Departamento de Engenharia Industrial, Pontifícia Universidade Católica do Rio de Janeiro.

The present work has as objective to analyze the investment of a XTL plant using the Real Options Theory. This methodology is adjusted to evaluate the capacity that this project has to changes its input and/or output, in other words, to elect the option that maximizes payoff, in accordance with each scenario, allowing to evaluate the construction of a plant with flexibilities with the investments happening in an environment of economical and/or technical uncertainties, where the prices (uncertainty factors) are stochastic and will follow the Mean Reversion Model, calculated by the Monte Carlo Simulation. The XTL technology is divided in two stages: a gasification process followed by a GTL (gas-to-liquid) process. The gasification process allows you to transform solids, liquids and gases into synthesis gas, that will be used as input of the GLT. The GTL makes possible to transform the synthesis gas into high quality liquids, such as naphtha, diesel, paraffins and lubricants. This work is part of a project, with the objective to apply the Real Options Theory in the evaluation of investments in Research and Development $(\mathrm{R} \& \mathrm{D})$, aiming to price and consider the flexibilities that are inherent to this project.

\section{Keywords}

Project Valuation; Real Options; Mean Reversion Model. 


\section{Sumário}

1 Introdução 14

1.1.1. Posicionamento da Dissertação 16

2 Análise de Investimentos: Método Clássico X TOR 21

$\begin{array}{ll}\text { 2.1. Investimento } & 21\end{array}$

2.2. Método de Avaliação de Investimento Tradicional - FCD 23

2.3. Teoria das Opções Reais - TOR 24

2.3.1. Opção 24

2.3.2. Evolução Histórica das OR 26

2.3.3. Abordagem da TOR 28

2.3.4. Tipos de Opções 30

2.3.4.1. Opção de Espera 30

2.3.4.2. Opção de Expansão 31

2.3.4.3. Opção de Redução 31

2.3.4.4. Opção de Paralisação Temporária das Operações 32

2.3.4.5. Opção de Abandono 32

2.3.4.6. Opção de Troca de Input / output 32

2.3.4.7. Opção de Investimento em Informação 33

2.3.4.8. Interação entre Opções 33

2.3.5. Considerações Finais $\quad 34$

3 Base Teórica 36

3.1. Processos Estocásticos 36

3.1.1. Processo de Markov 37

3.1.2. Caminho Aleatório 38

3.1.3. Processo de Wiener 39

3.1.4. Processo Generalizado de Wiener ou Processo Aritmético 40

3.1.5. Processo de Itô ou Processo Browniano Generalizado 41

3.1.6. Movimento Geométrico Browniano (MGB) 42

3.1.7. Movimento de Reversão à Média (MRM) 43

3.1.8. Processo de Poisson 46 
3.1.9. Lema de ltô 48

3.2. Técnicas de Otimização Dinâmica sob Incerteza 49

3.2.1. Programação Dinâmica 49

3.2.2. Direitos Contingenciais 51

3.3. Método de Avaliação de Opções 52

3.3.1. Modelo Binomial 52

3.3.2. Simulação de Monte Carlo (SMC) 54

4 Tecnologia XTL $\quad 59$

4.1. O Processo do XTL 62

4.1.1. Estágios da Tecnologia 63

4.1.1.1. Geração do Gás de síntese 63

4.1.1.2. Processo de Fischer-Tropsch 65

4.1.1.3. Hidroprocessamento 67

4.1.1.4. Gaseificação 68

4.2. Benefícios da Tecnologia XTL 71

4.3. Os Principais Players do Mercado XTL 75

4.4. Plantas XTL 78

4.4.1. Bintulu na Malásia $\quad 79$

4.4.2. Plantas da SASOL 80

4.4.3. Plantas no Qatar 81

4.4.4. Estudos de Plantas na América Latina 83

4.4.5. Estudos de Plantas no Brasil 84

4.4.6. Futuras Plantas no Mundo 85

5 ESTUDO DE CASO 86

5.1. Características do Projeto 86

5.1.1. Custos da Planta GTL 86

5.1.1.1. Investimento - CAPEX 88

5.1.1.2. Custos Operacionais 90

5.1.1.3. Custos do Gás Natural 91

5.1.2. Custos das Plantas BTL, OTL e RTL 91

5.1.3. Receitas das Plantas XTL 92

5.1.4. Rendimento dos inputs 94 
5.1.5. Outras Premissas do Projeto 94

5.2. Projeto XTL: Caso Particular de uma Planta de 35.000 Bpd 95

5.3. Cálculo dos Parâmetros das Séries de Preços 96

5.3.1. Cálculo Via Otimização 100

5.3.1.1. Teoria Darwiniana 101

5.3.1.2. Algoritmos Genéticos 102

5.3.2. Cálculo Via Regressão 103

5.3.3. Escolha e Análise dos Parâmetros 108

5.4. Simulação do Processo de Reversão à Média 109

6 Resultados 112

6.1. Análise de Sensibilidade $\quad 120$

6.1.1. Números de Iterações $\quad 120$

6.1.2. Correlações 122

6.1.3. Custos Operacionais 123

6.1.4. Investimento - CAPEX 125

6.1.5. Preços dos inputs 128

6.1.6. Preços dos Outputs 130

6.1.7. Perfil de Produção 133

7 Conclusões 135

8 Bibliografia 138

Apêndice A: Conversões das Séries de Preços 144

Apêndice B: SMC para MRM real e neutro ao risco 145 


\section{Lista de figuras}

Figura 1 - Valoração da Flexibilidade Gerencial 30

Figura 2 - Processo Generalizado de Wiener 41

Figura 3 - Variância Crescente de um MGB 43

Figura 4-MRM com tendência 45

$\begin{array}{ll}\text { Figura 5 - Mercado de petróleo com saltos } & 47\end{array}$

Figura 6 - Árvore Binomial em três passos $\quad 54$

Figura 7 - Funcionamento da SMC $\quad 56$

Figura 8 - Esquemático das etapas do processo do XTL 61

Figura 9 - Esquema de Gaseificação da RAUDI 69

Figura 10 - Tecnologia GTL da planta ORYX 83

Figura 11 - Custos do GTL (US\$/bbl) 89

Figura 12 - Divisão do CAPEX 89

Figura 13 - Divisão do CAPEX para plantas de BTL, OTL e RTL 91

Figura 14 - Distribuição de Anderson-Schulz-Flory 93

Figura 15 - Preço do Gás Natural 97

Figura 16 - Preço do Óleo Pesado 97

Figura 17 - Preço da Nafta 98

Figura 18 - Preço do Diesel 98

Figura 19 - Preço de Lubrificante 99

Figura 20 - Preço da Parafina 99

Figura 21 - Regressão para o Óleo Pesado 105

Figura 22 - Regressão para o Gás Natural 106

Figura 23 - Regressão para o Diesel 106

Figura 24 - Regressão para a Nafta 106

Figura 25 - Regressão para a Parafina 107

Figura 26 - Regressão para Lubrificante 107

Figura 27 - Simulação Real e Neutra ao Risco para o preço do GN 111

Figura 28 - Caminhos do MRM para o Gás Natural 112

Figura 29 - Caminhos do MRM para o Óleo Pesado 113

Figura 30 - Caminhos do MRM para a Nafta 113

Figura 31 - Caminhos do MRM para o Diesel 114

Figura 32 - Caminhos do MRM para o Lubrificante 114 
Figura 33 - Caminhos do MRM para a Parafina 115

Figura 34 - Histograma do VPL fixo Usando só GN 117

Figura 35 - Histograma do VPL fixo Usando só OP 118

Figura 36 - Histograma do VPL fixo com opção de input 118

Figura 37 - Histograma do VPL com opção de output usando GN 119

Figura 38 - Histograma do VPL com opção de output usando OP 119

Figura 39 - Histograma do VPL com opção de input e output 120

Figura 40 - VPL's para diferentes números de iterações_01 121

Figura 41 - VPL`s para diferentes números de iterações_02 121

Figura 42 - VPL's para diferentes correlações entre os inputs e outputs 122

Figura 43 - Aumento do OPEX para plantas com flexibilidade de input_01 123

Figura 44 - Aumento do OPEX para plantas com flexibilidade de input_02 123

Figura 45 - Aumento do OPEX para plantas com flexibilidade de output 124

Figura 46 - Variações no CAPEX das plantas GTL_01 126

Figura 47 - Variações no CAPEX das plantas GTL_02 126

Figura 48 - Variações no CAPEX da Planta OTL_01 127

Figura 49 - Variações no CAPEX da Planta OTL_01 127

Figura 50 - Variações percentuais nos preços do GN 128

Figura 51 - Variações Percentuais no rendimento do GN 129

Figura 52 - Variações no Preço do Óleo Pesado frente ao Petróleo 129

Figura 53 - Variações Percentuais no Rendimento do Óleo Pesado 130

Figura 54 - Variações no preço do Lubrificante 131

Figura 55 - Variações no Preço da Parafina 131

Figura 56 - VPL's para diferentes perfis de produção 134 


\section{Lista de tabelas}

Tabela 1 - Comparando opção financeira de compra e opção de investimento real

Tabela 2 - Classificação das Opções

Tabela 3 - Composição do GS da biomassa -Vol. Mol/mol (\%)

Tabela 4 - Comparação de Combustíveis

Tabela 5 - Projetos industriais em Operação

Tabela 6 - Estimativa de Custos de Investimento por Perfil de Escala

Tabela 7 - Rendimentos Estimados para diferentes matérias-primas

Tabela 8 - Alíquotas de Impostos e Taxas relevantes

Tabela 9 - Parâmetros Estimados

Tabela 10 - Parâmetros Via Regressão das séries originais

104

Tabela 11 - Parâmetros Via Regressão das séries convertidas

105

Tabela 12 - Parâmetros Utilizados

Tabela 13 - Correlações

Tabela 14 - VPL's Encontrados

Tabela 15 - VPL's para diferentes OPEX

Tabela 16 - Proporções Restritas para os Outputs

Tabela 17 - VPL's com proporções restritas para outputs

Tabela 18 - VPL's com proporções restritas para outputs e diferente preço para Lubrificante 